\title{
Computer-Integrated Construction: Background and a Case Project
}

\author{
Luca Caneparo
}

\begin{abstract}
Computer-Integrated Construction (CIC) is the capability of a computer system to create models of certain aspects of the design and construction processes and consequently to direct them into reality.

The premise of CIC is that the clear-cut separation between the competencies, know-how and responsibilities has now been turned upside down by the introduction of information technology in the design and construction processes. The designers can now model the physical, technological, and organisational aspects of a building or infrastructure in a seamless way. And they can do so rather creatively.
\end{abstract}

The model of the physical system provides the designer an intuitive understanding and appreciation of static and dynamic interaction between the building and its environment early during design.

The model of the technological system opens the capability to precisely model the building parts "as-constructed" by means of quantitative and qualitative-visual evaluation before any manufacturing process really takes place. The paper briefly introduces CIC in four construction technologies, respectively concrete, stone, wood and steel.

The model of the construction organization is the process of configuring an organisational structure to accomplish a given high-level task while attempting to satisfy stated performance objectives. An organization model represents people supported by information-processing and communication tools.

The CIC implementation and on-field experimentation is considered in a case project.

Index Terms - computer-integrated construction, project modelling, glued laminated timber, computer numeric control, distributed collaboration, concurrent design.

\section{INTRODUCTION}

\section{omputer-Integrated Construction (CIC) is the capability}

of a computer system to create models of the certain aspects of the construction process with the facility to direct them into reality.

The premise of CIC is that the clear-cut separation between the competencies, know-how and responsibilities has now been turned upside down by the introduction of information technology in AEC. Designers can now model the physical

Luca Caneparo is with Politecnico di Torino, Associate Director of Laboratorio Alta Qualità - Territorial Integrated Project, (phone: 390112276 924, fax: 0112276 929, e-mail: luca.caneparo@polito.it). specifications, the manufacture and organisational aspects of a building or infrastructure in a seamlessly. And they can do so rather creatively.

Every computer-aided design system used in AEC

incorporates assumptions about construction. The aim of CIC is to shift from implicit assumptions towards explicit models of the construction process and to create the building or infrastructure according to the templates dictated by the models. From a philosophical point of view, the property of CIC is a system's evolution from being descriptive to being prescriptive.

Until the introduction of digital computers, analog models, i.e. mock-ups, structural and energy models were the main aspects of buildings modelled. Nowadays computers can model and predict the physical aspects of a building with finer tolerances than can actually be constructed [7], with detail and evidence of manufacture and organisational aspects, which otherwise can not experienced directly or defined in a direct cause-effect nexus because of complexity. But most importantly, the CIC models are dynamic-interactive: the power of computers and software allows us to understand and evaluate the interrelation of a design issue with all the aspects modelled in the system. CIC differs from physical based simulation systems in that it has the potential to direct the model to become real, modelling technological and organisational aspects. CIC differs from CNC in that the scale of most buildings is too large to be created directly by means of NC. It is not only a matter of scale, most buildings are complex systems where several issues, architectural, technological, structural, managerial, organisational, constructive etc. interact at the same time. CIC implements aspects common to both Product Data Management (PDM) and Enterprise Resource Planning (ERP) technologies. Basically, an ERP implementation is integrated around a unitary data model oriented to the coordination of various applications aimed to production, e.g. scheduling, planning, control [9,41,46]. PDM is more focused on integration of independent applications, frequently developed by different vendors and running on diverse systems. CIC relies on a close, interactive, integration between various modelling and simulation tools.

Construction is a compound process, which requires both human and machine interaction and coordination. CIC monitors this interaction, and is able to change the models in order to cope with unplanned or random events.

Because of the number of the building typologies and of the construction systems presently in use, it is impossible to define the model capabilities of CIC univocally: it requires 
several coexistent representations-models of the construction process, which are defined by three dimensions, respectively the physical, technological and organisational.

\section{A. Model of the Physical System}

Most CAD systems are geometry-oriented, while to model the physical aspects of a building, much more information on the materials and their physical specifications, the environment and the building process is required. A shape is modelled differently depending upon the aspects considered in the design at the time. For example, the requirements in curvature, bending, torsion, angle etc. differ according to whether the shape is to the construction technology (e.g. wood, stone, iron, steel or concrete) by means of a milling, cutting, reforming, molding or casting process. To account for these requirements, CAD solid or surface models are required to integrate the geometry descriptions with the physical specifications. The modelling of the physical system is considered from the point of view of requirements, constraints and capabilities posed respectively by the design of the shape and the specifications of the material.

\section{1) Shape-Oriented Modelling}

Continuity or discontinuity of the surface is an effective property of several materials and manufacturing processes. To evaluate the quality of curves, tools assign colours based on the curvature value, or render images using colour and texture to simulate highlights or neon row with the capability to dynamically change the number, spacing and orientation of the lights to obtain the best surface evaluation $[17,37]$. In analytical terms the continuity can be defined as the derivative of the curve. First-derivative continuity, defined as slope, assures continuity of the tangent vector at vertices. In second-derivative continuity, defined as curvature, the slope and the derivative of the slope are continuous along the curve. The analytical evaluation of a shape can be refined further. " $\mathrm{A}$ curve is fair if its curvature plot is continuous and consists of only a few monotone pieces" [14]:

$$
\frac{\mathrm{d} k}{\mathrm{~d} s}=\frac{\operatorname{det}[\dot{x}, \dddot{x}]}{\|\dot{x}\|^{4}}-3 \dot{x} \ddot{x} \frac{\operatorname{det}[\dot{x}, \ddot{x}]}{\|\dot{x}\|^{6}}
$$

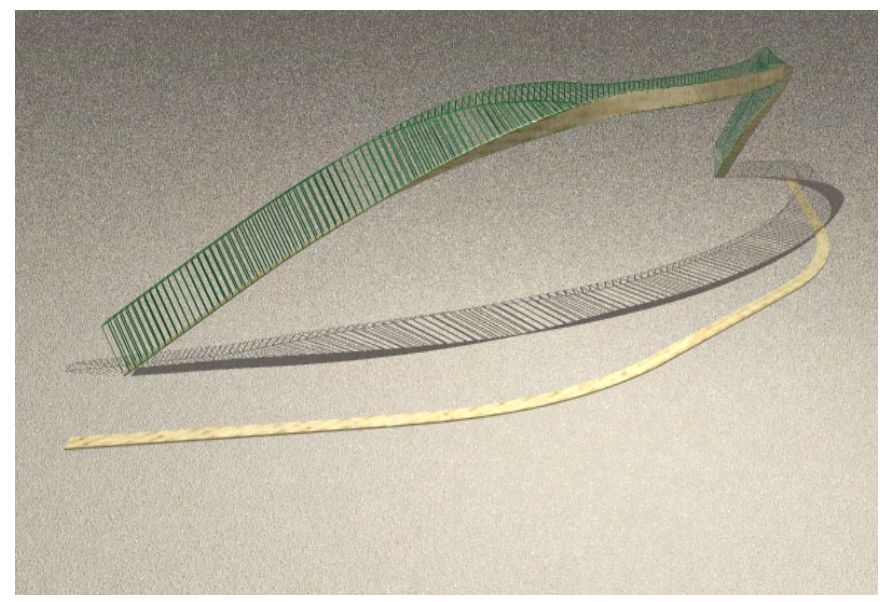

Figure 1. Material-Oriented Modelling. where $k$ is the slope, $s$ is the arc length and dots represent derivatives with respect to the given parameter $u$ of $x(u)$. Several CAD systems implement tools to evaluate the degree of continuity. These can be post-process or interactive tools. If interactive, during modelling, wireframe, facets or normal vectors are highlighted with colours according to the derivative degree of continuity $[2,10,18]$. Further analytical tools have been developed to evaluate the degree of continuity $[2,14]$.

Shape-oriented modelling system, integrating tools to analyse and represent the continuity of a shape, provides the designer coexisting point of views on aesthetic, structural and constructive aspects. From an aesthetic point of view, these tools aid to pursue either stability or instability of the form. They are an analytical aid to the visual evaluation, which in certain designs can demonstrate difficult or partial. From a structural point of view, they show curvature extrema or points where the bend changes suddenly, e.g. flat spots or saddle points. They provide a qualitative-visual aid to interpret continuity in torsion and curvature along the form. In the view of construction, these tools prevent even small discontinuities, e.g. inflections, which certain materialtechnology requires specific procedure.

\section{2) Material-Oriented Modelling}

In Material-Oriented Modelling (MOM) as the designer refines the models, i.e. outlines the materials and the loads conditions associated to the shape, the computer system refines the simulation of the behaviour of the form. It is not a post-simulation, where the designer passes the model to an analytical tool, e.g. Finite Elements Analysis (FEA). It can be much more interactive, because the simulation tool uses the same model of the CAD system, without a meshing process [3,5]. Furthermore the meshless analysis proves up to order of magnitude faster than $\mathrm{P}$ - and H-element solvers of FEA.

For example, if the designer assigns to a shape the specifications of a metal material, the system visualises areas inside and outside the elastic range of deformation. The visualisation can be in stop colours -red, yellow and green- to allow designers addressing first-level analysis in an intuitive way during the modelling process. Green means that the designer can proceed. Yellow signals that further cautions are required, e.g. more detailed design, analysis or the interaction with a specialist. Red means that it is necessary to rethink the design.

As the designer interacts with the shape, the loads, or details the materials properties in a hierarchy of materials models to yield the desired one [11,28,43], the system updates the simulation and visualisation. A more detailed description allows the system to enlarge the range of confidence of the model towards more precise representation (Figure 1). MOM is not conceived to substitute FEA, which provides a higher level of detail and accuracy. Even though the MOM can provide a measure of expected error and confidence on the simulation, its purpose is to lead towards an intuitive understanding and appreciation of static and dynamic interaction between the building and its environment early during design. Simplifying the model preparation, the meshless analysis is also useful in problems with changing 
domains, such as crack propagation, where FEA experiences difficulties in following the changing domain.

\section{B. Model of the Construction Technology}

The Model of the Construction Technology (MOCT) is seamlessly integrated with MOM, of which it constitutes a further level of detail. The designer directs the form more precisely towards a construction technology, and benefits from the integration of MOCT with MOM in that s/he acquires direct control of the process, which is not only descriptive, drafting-based, but becomes prescriptive as well. It turns into descriptive, because NC manufacturing allows the form to be created with little or no further human mediated intervention. This certainly challenges the capabilities and know-how of the designer, who acquires both greater control and responsibility. Without doubt several human mediated interventions along the building process amplify the possibility of misunderstanding, whereas they give an opportunity to refine-correct the execution of the design. In NC manufacturing an error or unforeseen aspect can propagate along all the process quickly, hence the relevance of modelling the building consistently increases.

CIC integration opens the capability to model the building parts "as-constructed" precisely by means of quantitative and qualitative-visual evaluation before any manufacturing process really takes place. Because in the building trade no unique construction technology exists, but several different ones are feasible, the paper briefly introduces three: tooling, forming and casting-molding.

1) Tooling of Stone, Wood, Plastic and Metal

Tool refers to the manufacturing process where a computercontrolled machine moves the tool and/or the object along a programmed path. Machines and tools exist to cut-remove a wide range of materials: stone, wood, plastic, metal etc. The machines are usually grouped according to the movement they can impress to the tool-part: from two axes up to five.
The capabilities of tool manufacturing are becoming much more widely available to the designer with the integration of CAM systems with material specifications such as strength, density, texture and finish, sometimes inherited from MOM [32] (Figure 2).

The software is increasingly able to profit the integration of the geometry and material descriptions to automatically recognise and optimise paths, directions, movements, speed, tangency, roughing, finishing and eventually change of the tool $[8,13,20,44]$.

Closer integration of MOM with MOCT provides the designer with tools to highlight differences between the "as-machined" vs. the modelled object, before the NC data are transferred to the factory floor. The in-process simulation can quantify the accuracy of the "as-machined" part considering aspects relating form (e.g. gouges, clashes, under- or over-cuts), the material and the tool path. False colour visualisations highlight dimensional variance due to the tooling and any material remaining. Realistic CG animation of the tool path is useful for evaluating quality, optimisation strategies, roughing options, surface finishing, tolerances and possible collisions of the tool $[12,36,44,45]$.

To minimise the waste of valuable materials, the software can automatically define the optimal assignment of the parts to be manufactured to the raw blocks. This option can demonstrate cost effective especially with a great number of parts or in the case of blocks of irregular shape, e.g. wood, stone.

2) Forming of Metal, Glass, Wood and Polymer A process of application of loads-forces and/or heating can reform several materials (e.g. metal, glass, wood and polymer).

The importance of the close integration of MOM with MOCT lies in the assistance to the designer in modelling shapes coherent with both the physical and manufacturing reality. Material-Oriented Modelling of curved or multi-curved surfaces considers texture, elastic or plastic constraints of the
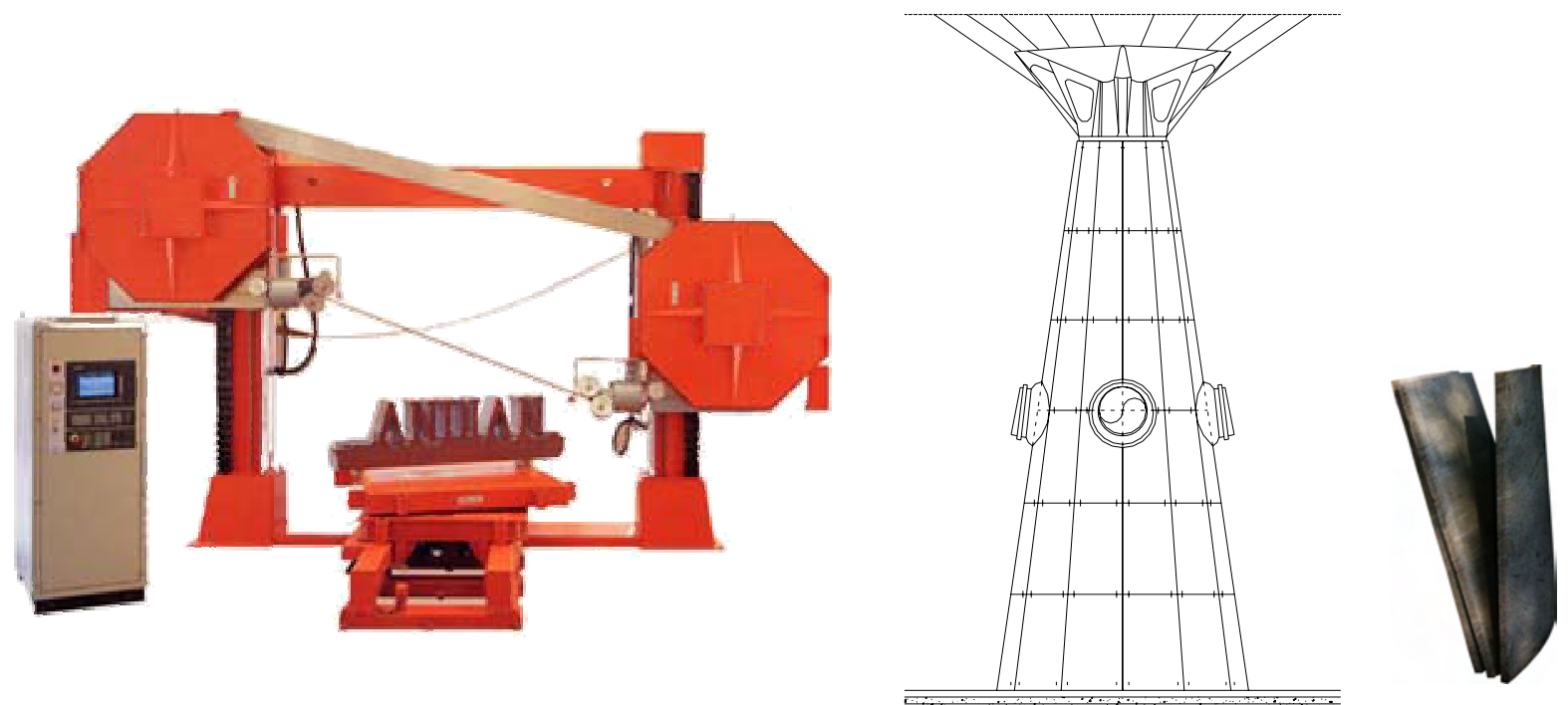

Figure 2. CNC cutting of granite slabs, columns of Kuala Lumpur airport. 


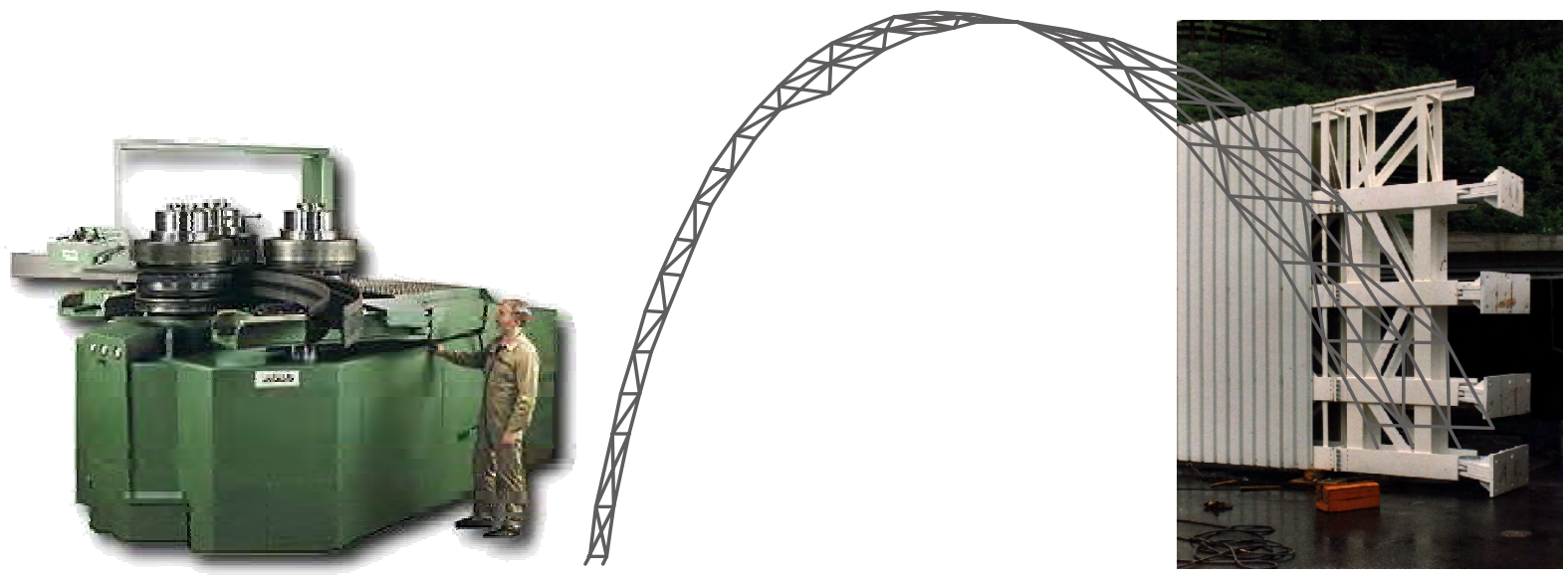

Figure 3. CNC bending of T beams, gateway access to Courmayeur tunnel.

material. Since the constraints of the material are satisfied during the design process, the software can automatically develop the designed surface in the plane, before applying bend and torsion. The profile of the curve in the plane is manufactured either as a whole or parts by means of NC. The software interprets the material and bend parameters as NC instructions for the machine, which forms the overall object or each part to the calculated curvature (Figure 3).

3) Casting of Concrete - Molding of Metal and Plastic In principle any material with a fluid state can be used for a casting/injection process during which it acquires the shape of the mold. In the case of material with quick solidification, high cohesion, relevant retraction or objects with thin parts, the designer can significantly benefit from a design system integrating the shape requirements with those of the casting/injection process and the characteristics of the material.

The flow analysis is becoming an integral aspect of the design process since it can suggest modification to the shape and to the parameters to fill the mold, e.g. pressure, vibration, temperature, points of injection $[34,35,38]$.

The art of casting/injecting relies also on the art of mold making. It is important -apart from the other general characteristics of a good mold- that there should be no dielock parts, which will cause problems during the extraction of the object. Shape-evaluation tools are integrated in the system to visualise dielock conditions from a defined pull direction during design. To evaluate the surface specifications of a mold, e.g. roughing, finishing and tolerances, designers can benefit from NC simulation, before the mold is manufactured. In the case of formworks, for example for casting concrete [21,22], the designer can use forming technologies to create the mold, including multi-curved surfaces, from the computer model.

\section{Model of the Construction Organization}

Since most buildings are simply too large to be manufactured as a single piece, the construction process has to deal with a widely distributed organization, which concludes sometime at the construction-site.
The paper does not explicitly consider highly automated processes, where NC machines and robots integration play a primary role in both manufactory and on-site construction [39]. Conversely it deals with the ongoing spreading of NC technologies in industries manufacturing materials and components for the construction trade. Present investments in CAD/CAM and NC technologies will multiply their benefits if they extend behind the AEC firms, design offices and factory floor (MOM and MOCT) towards the whole construction process.

While MOM and MOCT deal with the physical and manufacturing aspects of the construction process, the Model of the Construction Organization (MCO) is oriented to the modelling of the managerial aspects of the project. Organisational modelling is "the process of configuring an organisational structure to accomplish a given high-level task while attempting to satisfy stated performance objectives. An organization includes people supported by informationprocessing and communication tools.” [29]. The background of MCO dates back to Weber's theory and successive development by Simon [31,42]. Galbraith [19] and Levitt $[29,30]$ information processing approaches to organization lead the way to model and simulate work processes.

The Workflow Process Definition Language (WPDL) and IDEF3 are two promising languages for representing work processes. The WPDL [23] was developed by the Workflow Management Coalition, a non-profit international organization of workflow vendors, users and analysts, founded in 1993. IDEF3 was developed by the U.S. Air Force to standardise a technique for stating requirements [33]. IDEF3 compared with WPDL has a more detailed representation of the work, since it explicitly represents the individuals, machines and resources participating to the process.

One aim of modelling construction organizations is the analysis, namely the understanding of the specific way they perform the work, their strong and weak points. After comes the capability to simulate scenarios, i.e. designing different structures of the organization to evaluate the benefits in terms of time, quality and cost $[6,29]$. 
A further aim of modelling a construction organization, either an existing or planned one, is its operative implementation in a PDM or ERP system. Research issues are open to system integration of modelling the relations between the actors, the tasks and the resources of construction processes and the relevant product model schemas [27]. At present there are promising applications arising from the integration of implementation independent languages, such as WPDL and IDEF3, and ERP systems $[1,4,15]$. This integration can lead three major benefits for the construction industry: Speed, the concept is based on a short and compact implementation cycle, minimising the implementation effort through the use of standard, well recognised, tools. Flexibility, using standard tools, construction companies can adapt ERP or PDM systems to the changing organisational structures, construction practices, and onsite procedures. Reliability, the use of standard tools can drive both ERP system developers and third-party developers in working out and supporting construction industry-specific models that let companies take full advantage of widely-accepted and proven procedures and methods.

\section{Model of Construction Materials Management}

The Model of Construction Materials Management (CMM) serves to represent and therefore optimise the flow of materials and components during the construction process. The CMM belongs to the organisational, management and production activities, from the raw materials to the components, from the sources through the work processes to the site. The role of CMM in the computer-integrated construction process is particularly important, because it shifts building tasks and processes from the construction site to manufactory: CIC tends to produce value-added components, which are to assembled onsite.

A crucial aspect of CIC is the coherence of the models with the construction process along the different phases and aspects. Before spread of the digital computer, organization and management models had hardly been considered as subject of experimentation: they were more a subject of validation by means of statistical observations. MCO and CMM models can be validated and increase their effectiveness through continuous monitoring of the construction process in order to map its progress and unforeseen or unpredictable events. For researchers the monitoring provides data to refine the models and systems, while it offers the construction industry working tools to improve the total quality management, to reduce the time cycle and to optimise the use of resources. Effective monitoring technologies to be integrated with CIC are:

Total station instruments are habitually used on-site to locate and survey precisely. They are interfacable to computer systems to provide spatial coordinates in digital format. However their use is demanding and lengthy: they require a trained operator with, in certain cases, an assistant to manually identify and then survey each object.

Barcode is a robust technology widely used. It is based on a labelling system to be attached to each item to identify it univocally. It requires an untrained operator to point a laser hand-held device to automatically read the label. It requires both line of sight and proximity between the reader and the label. Barcode technology makes it possible to identify objects throughout the construction process, from the factory to the construction-site, but surveying of the items is provided only indirectly either by a fixed reading station or by the operator measuring with further technologies.

Radio frequency identification (RFID) is rapidly emerging as a promising technology because it does not require a line of sight and the proximity is extending to the range of ten of meters [16]. It is based on either a passive or an active transponder and a fixed/mobile RF station. The mobile station is comparable in size and weight to a handheld barcode reader. When the transponder receives a signal from the station it starts a read/write communication session. The transponders have onboard read and/or write memory up to several $\mathrm{Kb}$ (Figure 4). Each transponder is identified by a unique digital code. The transponder $\mathrm{r} / \mathrm{w}$ memory can be used to store information on the associated item along all the construction process. On the factory floor the memory can carry information on the manufacturing cycle as well as exceptions or peculiarities, e.g. tooling, finishing etc. At the construction-site or later during the building maintenance, it can store information of tasks completed, in progress or scheduled.

\section{E. A Case Project}

Close to Aosta, Italy, it was decided to build a protective structure at the intersection between the motorway TurinCourmayeur and the cableway to Pila. The structure protects vehicles on the motorway from the eventuality of a falling cabin or, more likely, from objects and blocks of snow, which might fall down from the cableway above it.

Four arcs span the motorway and sustain a grid of security glass over the four ways lanes. Each arc measures 48 metres, and is curved, along the main axis, to magnify the impression of entrance, driving along the motorway (Figure 5, 6). The location of the structure, close to the city centre of Aosta, could even acquire the significance of gateway - entrance to the city. The architectural design communicating this symbolic meaning.

The dimensions of the structure are constrained by, on the lower side, the maximum shape of the vehicles on the motorway and, on the upper side, by security clearance of the cableway. To meet with these constraints, the two arcs

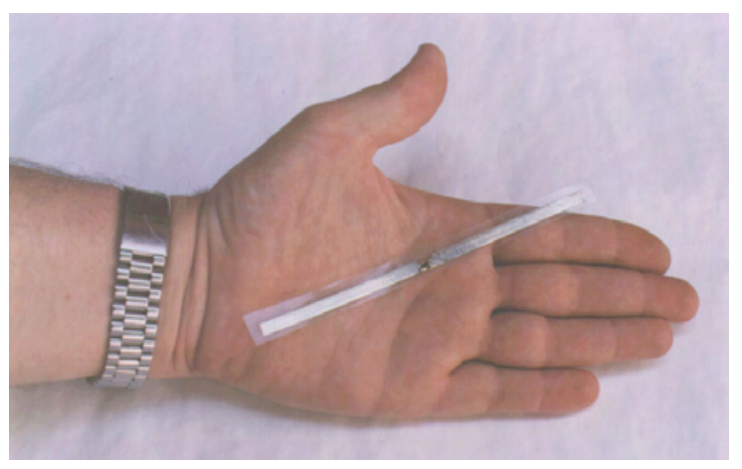

Figure 4. A radio frequency identification transponder. 

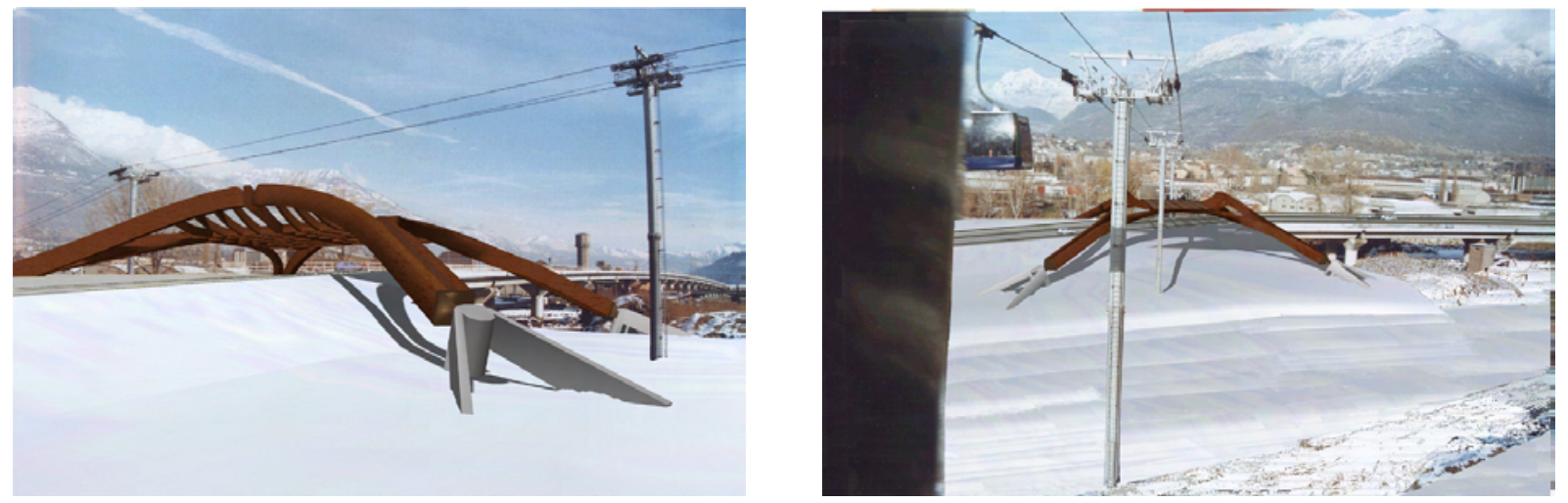

Figure 5, 6. Early computer renderings used to obtain the contract.

crossing the cableway are torsioned along their axes, so that in the middle they are horizontal, i.e. $90^{\circ}$ degrees torsioned. The motorway was to be inaugurated, so there was less than a year ahead to complete the structure, starting from design. Due to the tight schedule the customer was especially motivated to implement a CIC system with the aim to develop the project concurrently.

\section{1) CIC Implementation}

Seamless integration between MOM, MOCT, MCO and CMM was pursued embedding the applications within a modelling kernel or, when this was unfeasible, by means of a three tiers-architecture.

The front-end tier was implemented by means of Microsoft's Component Object Model (COM), which seamlessly integrated at-runtime the capabilities of the applications. Microsoft's Distributed Component Object Model (DCOM) allowed objects residing in distributed applications to collaborate in a unified way by means of acknowledge protocols and specifications. The semantic interoperability between applications, collaborating via DCOM, was pursued by STEP or IFC standards $[24,25,26]$.

These standards defined the semantic on which the applications collaborated via the middle and back-end tiers. The middle tier is the project server, which stored the application data as projects. The back-end tier is the client/server distributed object oriented database. STEP and IFC semantic definitions with DCOM interoperability defined a dynamic data model: the modelled objects, new data structures or requirements to the models could be changed at run-time during the project.

2) Material-Oriented Model

The modelling of the timber beams posed shape, technological and structural requirements and constraints.

The shape of every section, perpendicular to the barycentric axis, had to be rectangular. Moreover, two beams had $90^{\text {th }}$ degree torsion along their axes: they started vertical, became horizontal in the middle, and terminated vertical again. During the design process visually evaluating a shape, which in reality spanned 48 meters, from a monitor or even a large plot turned out to be difficult. The fair analysis of the computer models of the beams proved useful to evidence curvature extrema or points where the bend changed suddenly in the early design phase.
MOM aided the design team in modelling the shapes of the four arcs according to the constraints of the construction technology, glulam timber. MOM fulfilled bend and torsion constraints of glulam along all the four arcs as well as the single timber leaves since design inception. In view of the assembly process, MOM made the designer attentive to even small discontinuities in the bend, e.g. inflections, which could generate additional stresses inside the timber. The curvature and torsion of the beams had to accomplish the elastic bending of the single timber leaves, which composed the global beam by means of the juxtaposition of the laminated glue.

MOM proved useful giving prompt ideas on the structural behaviour during design, while more lengthy FEA analyses were run to verify and refine the model.

3) Model of Timber Construction

MOCT made it possible to develop each modelled layer of timber in the plane, including the multi-curved torsioned beams. Figure 7 illustrates half of one torsioned beam; the two edges, at the base of the beam, are juxtaposed to their profiles developed in the plane. The triangles, between the two edges, were displayed to visualise and verify the meshing procedure. The system enabled the design team to analyse and visualise the exact shape of each timber leaf when flat; i.e. before torsioning and bending it to assemble the beam in space. This was especially useful in the early design phase, when the capability to exactly simulate the profile of all the timber layers suggested the possibility of unifying the profiles. The possibility of cutting and assembling a unified layer of timber instead of 16 slightly different ones for each beam was considered cost effective. In the case of the unified profile, the software simulated the maximum shift from the bottom to the top layer of timber in $8 \mathrm{~cm}$, along the $180 \mathrm{~cm}$ vertical axis of the beam (maximum tolerance $0.044 \%$ ) (Figure 8). These data were considered during the modelling of the structure.

The integration of MOM with MOCT supported the translation of the profiles of the timber layers, developed on a plane, into cutting parameters and paths, and then in the CNC instructions for the machine (Figure 9).

The cutting paths, scaled 1 to 20, were also used to generate the CNC code to machine scaled Masonite leaves. Using glue the scaled leaves were assembled to build the mock-up of the four beams and the central grid (Figure 10). The mock-up was the effective demonstration of the feasibility of the CIC 


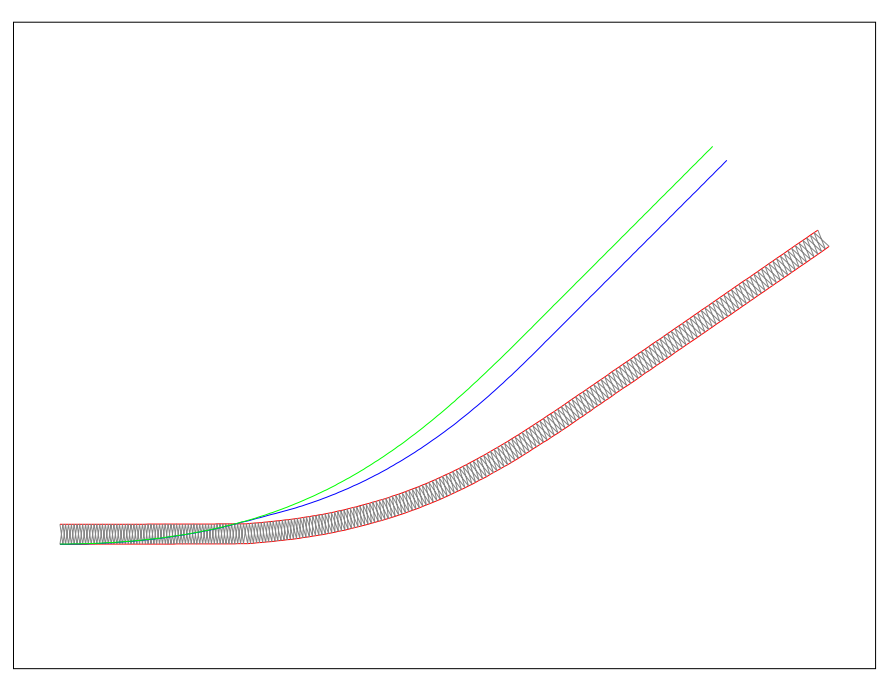

Figure 7. The edges of the base layer in the torsioned beam and developed in the plane.

methodology, that is the coherence between the models, the software and the physical reality.

\section{4) Models of Construction Organization and Material Management}

Usually, glue laminated structures are assembled over special bench press in a controlled humidity and temperature environment, which assures the creation of a composite material with predefined and well known specifications. The possibility of pre-assembling in the factory four arcs of 48 meters width and $10 \mathrm{~m}$ high and then shipping them for several kilometres to the construction-site was considered unfeasible. So pre-assembling the beams was discarded in favour of assembling them on-site. Assembling on site raised several problems.

A scaffolding was required to offer a temporary support for the layering and assembling of the timber leaves with the glue and screws.

Moreover, it was considered whether to pre-curve and torsion the timber leaves or not. Usually in glulam structures the timber leaves are formed to the final curvature and torsion keeping them for days in a high temperature and humid environment, i.e. oven, with applied constant or increasing bending and torsion forces. The absence in the beams of values outside elastic curvature and torsion suggested the possibility of forming the leaves directly on-site, over the iron scaffolding, without pre-curving them in an oven. FEA [47] and tests with full-scale leaves demonstrated that for 6 and 12 $\mathrm{cm}$ thickness of the leaves the glue and screws were sufficient to make and form the timber layers onsite.

Each timber leaf was uniquely identified using an RFID transponder. In the factory and at the construction-site mobile wireless LANs were established by means of RF Ethernet technology [40] connected to the CIC system via ISDN lines. On the shop floor fixed stations queried the transponders at the beginning and end of each major manufactory phase, e.g. cutting, finishing, stocking, shipping. The data to and from the transponders were communicated in real-time to the information system via the RF LAN.

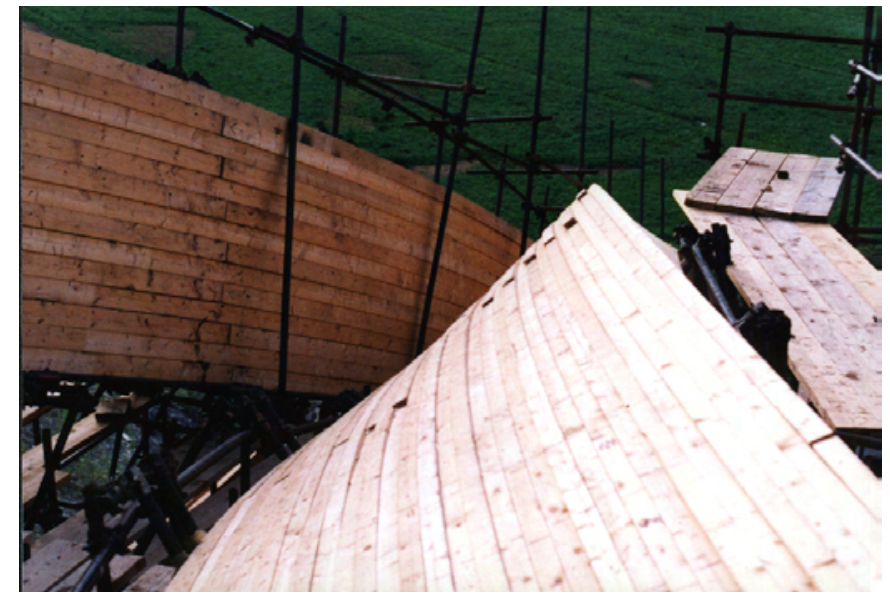

Figure 8. Two beams converging toward the bearing.

At the construction-site fixed stations at the gate and stocking area supported online check-in and check-out of the items. An operator with a handheld station could read/write data to/from the transponder and the CIC system directly.

The assembling of the structure on-site required both solid and exact shaping of the temporary scaffolding. The scaffolding was required to align the stacking of the leaves, to support the weight of the timber itself as well as the adjunctive forces generated during the forming of the leaves plus the tensions due to the consolidation of the glue.

About thirty templates (tubular metal squares of $70 \times 180 \mathrm{~cm}$ ) for each beam had to be precisely placed and rotated in 3D to align and support the stacking of the leaves (Figure 11). To place, align and verify the position of the templates and the leaves both total station and radio frequency identification technologies were used.

The RF LAN provided for online bi-directional communication between the total station and the integrated information system: the software of the total station downloaded the co-ordinates of the model from the information system directly. The site manager used these coordinate to exactly shape the temporary scaffolding, to position the thirty templates, which directly supported the timber layers. To accurate locate these points, the laser beam of the total station determined the axis to position the prism.

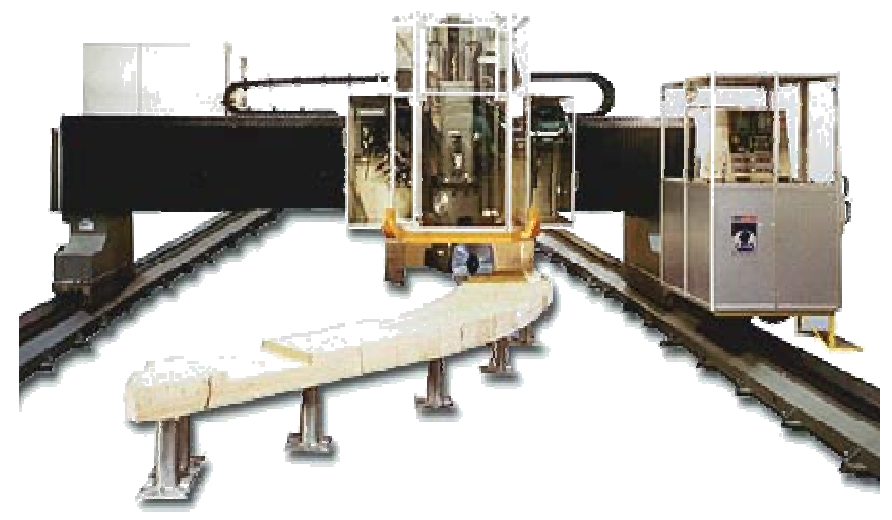

Figure 9. CNC machine to cut the leaves. 


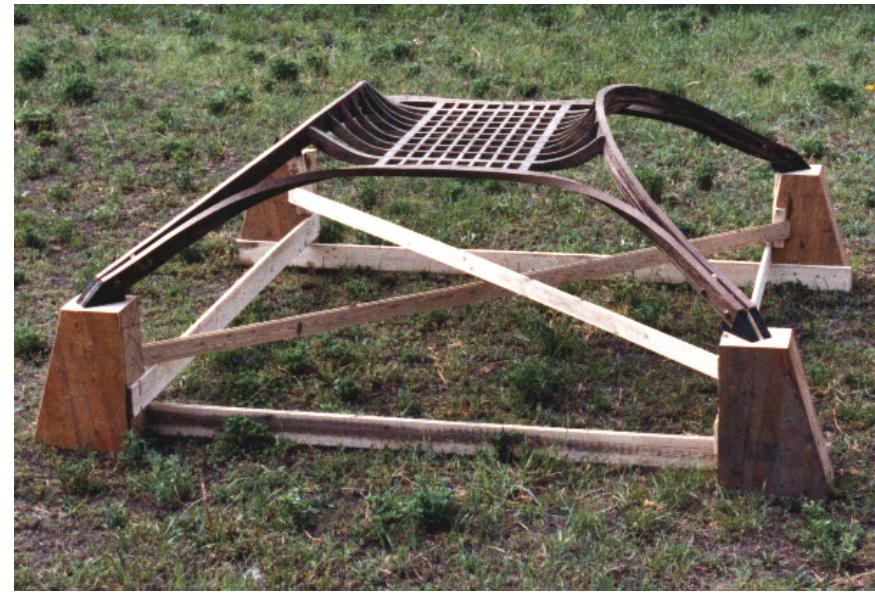

Figure 10. The 1:20 mock-up of the structure.

The rodman had to move the prism along the laser axis to fit the exact distance. The adjustments of the distance were based on the feedback from the surveyor at the total station. This process was simplified by the fact that the point lay at the intersection between a curve, the base edge of the beam, and the axis traced by the laser beam. So, often, only minor adjustments of the distance of the prism were required. Some total stations incorporate an assistant light changing colour or blinking cycle to indicate if the prism has to "go" or "come" to fit the exact distance automatically.

During the construction process the positioning of each timber leaf was surveyed in real-time into the CIC system. The surveying process was speeded up by the integration of total station and RFID technologies: the rodman identified an item by means of the handheld RFID station and then positioned the prism twice, respectively in correspondence with the lower-left and upper-right corners of the leaf, allowing the total station to get two measures and communicate them to the CIC system through the RF LAN. The system was able to associate these coordinates to the right leaf automatically, identifying the item by means of the unique ID of the transponder attached to it.

\section{F. Participants in the Project}

The main participants in the project were: Società Autostrade

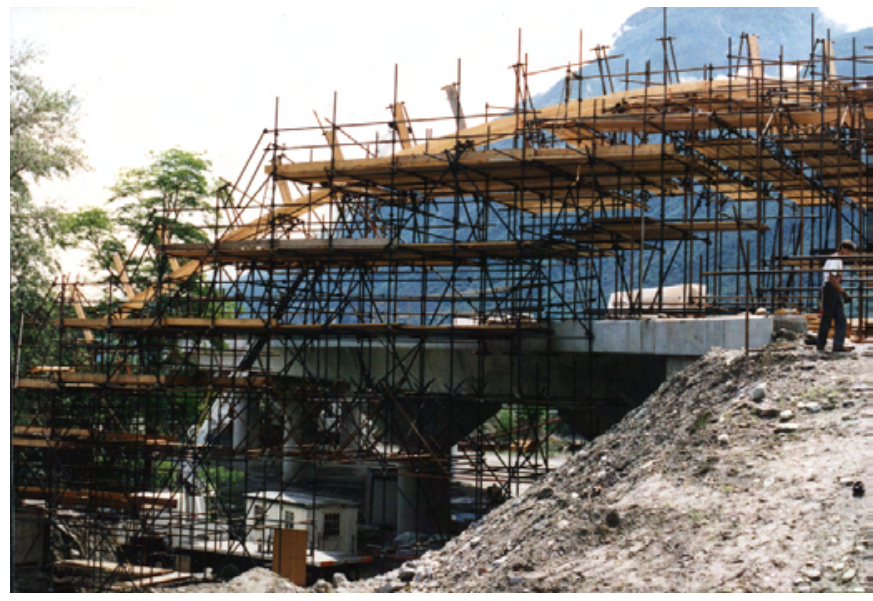

Figure 11. Temporary scaffolding with the templates and the first layers of timber.
Valdostane spa, customer; INCISA spa, mandatory; Arch. Sergio Beccarelli of Policreo, architectural design; Eng. Innocente Porrone, structural engineer; Arch. Luca Caneparo, IT manager; Chenevier spa, timber cutting and assembling; Edilchimica Italia srl, glue supplier.

\section{G. Conclusions}

As considered earlier in the case study, CIC was experimented during the design and construction of an infrastructure. CIC was shown effective in accomplishing the schedules and deadlines of the project. The integration of the different models reduced the overall time requested by the cycle of designing-manufacturing-transporting-assembling, and improved accuracy, and reduced tolerances. While the integrated design and management of the project suggested several possible optimisations, such as the unification of the profiles.

The real-time monitoring improved the project development towards a continuous process covering the whole models and aspects, beyond manufactory and construction, towards full interplay of information from designing to assembling and throughout competences and responsibilities. It made each model and phase of the project really interdependent and interrelated with each other. If, for example, a leaf did not fit with the designed position, because of settling of the structure, displacements due to glue tensions during the consolidation (Figure 12) or incidental adjustments of the scaffolding, the design team could model alternate solutions and evaluate the appropriate one. In certain cases, the solutions considered were a displacement of some leaves, different glue application or reshaping of some of the leaves still in the manufactory process.

\section{H. References}

[1] AcceleratedSAP. SAP AG, 1999: (http://www.sap.com/).

[2] Advanced Surface Extension. Pro/Engineer, 1999.

[3] Atluri, S.N. Zhu, T. "A new meshless local Petrov-Galerkin (MLPG) approach in computational mechanics”, Computational Mechanics 22

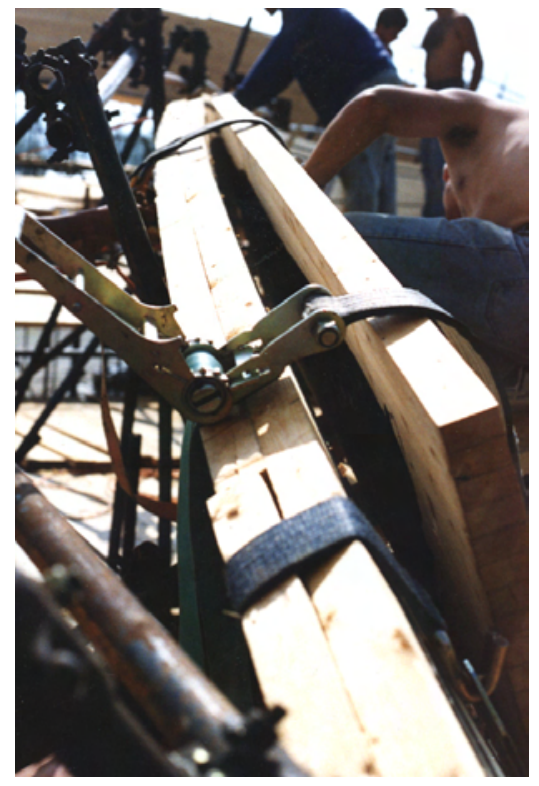

Figure 12. Stacking process of a timber leaf. 
(1998): 27-117.

[4] BaanWise and Dynamic Enterprise Modeling. Baan B.V., 1999: (http://www.baan.com/).

[5] Belytschko, T. Krongauz, Y. Organ, D. Fleming, M. "Meshless methods: an overview and recent development", Methods in Applied Mechanics and Engineering 139 (1996): 3-47.

[6] Butler, K. Esposito, C. Hebron, R. "Connecting the Design of Software to the Design of Work", Communication ACM 1 (1999): 38-46.

[7] Caneparo, L. "Simulation of shape, light, color and material in architectural design”, Colajanni, B. Pellitteri, G. (Eds.) Proceedings of ECAADE, Palermo: Arti Grafiche F.lli Giordano, 1995: 417-426.

[8] Catia MFG. CATIA V4R2, International Business Machines Corporation and Dassault Systèmes, 1998.

[9] Cook, M. Building Enterprise Information Architectures: Reengineering information systems. Upper Saddle River: Prentice Hall, 1996.

[10] “Curvature Properties”, Studio 9 Manual, Alias|Wavefront, 1999.

[11] Eberhardt, J.J. Hay, P.J. Carpenter, J.A. "Materials by design: a hierarchical approach to the design of new materials", Materials Research Society Symposium Proceedings 63 (1985): 191-206.

[12] ESPRIT. DP Technology, 2000: (http://www.dptechnology.com/).

[13] Expert Machinist. Pro/Engineer, 1999.

[14] Farin G. Curves and surfaces for computer aided geometric design: a practical guide. Boston: Academic Press, 1988.

[15] FastForward. Oracle,

1999: (http://www.oracle.com/fastforward/index.html).

[16] Finkenzeler, K. Radio-frequency identification fundamentals and applications, New York: Wiley, 1999.

[17] FreeStyle Optimizer. CATIA V4R2, International Business Machines Corporation and Dassault Systèmes, 1998.

[18] FreeStyle Shaper. CATIA V4R2, IBM Corporation and Dassault Systèmes, 1998.

[19] Galbraith, J.R. Organization design. Reading: Addison-Wesley Pub., 1977.

[20] Generative Machining. I-DEAS, SDRC, 1999.

[21] Hanna, A.S. Sanvido, V.E. "Interactive Vertical Formwork Selection", Concrete International: Design and Construction 12 (1990): 26-32.

[22] Hanna, A.S. Concrete formwork systems. New York: Dekker, 1999

[23] Hollingsworth, D. The Workflow Management Coalition Specification. Workflow Management Coalition Document No. TC000-1003, 1995.

[24] IAI. End User Guide to Industry Foundation Classes, Enabling Interoperability in the $A E C / F M$ Industry. International Alliance for Interoperability, 1996

[25] IAI. Industry Foundation Classes - Release 2.0 Specifications, IFC Object Models for AEC Projects. International Alliance for
Interoperability, 1998.

[26] ISO. Industry Automation Systems-Product Information Representation and Exchange - Part 1, Overview and Fundamental Principles. ISO TC184/SC4 Project Management Advisory Group, 1994.

[27] Junge, R. Kothe, M. Schulz, K. Zarli, A. Bakkeren, W. "The VEGA Platform”, Junge, R. (Ed.) Proceedings of CAAD Futures '97. Dordrecht: Kluwer Scientific Publishing, 1997: 591-616.

[28] Kaljevic, I. Saigal, S. "An Improved Element Free Galerkin Formulation”, International Journal for Numerical Methods in Engineering 40 (1997): 2953-2974.

[29] Kunz, J.C. Christiansen, T. Cohen, G.P. Jin, Y. Levitt, R.E. "The Design Virtual Team”, Communication ACM 11 (1998): 84-91.

[30] Levitt, R.E. Cohen, G.P. Kunz, J.C. Nass, C.I. Christiansen, T. Jin, Y. "The Design Virtual Team”, Carley, K.M. Prietula, M.J. (Eds.) Computational organization theory. Hillsdale: Lawrence Erlbaum Associates, 1994.

[31] March, J.G. Simon, H.A. Organizations. New York: Wiley \& sons, 1958.

[32] Material Data System. I-DEAS, SDRC, 1999.

[33] Mayer, R. Cullinane, T. deWitte, P. Perakath, B. Wells, M. Information Integration for Concurrent Engineering (IICE): The IDEF3 Process Description Capture Method Report. USAF Armstrong Lab, 1992.

[34] Moldflow, 1999: (http://www.moldflow.com/).

[35] MPI/Flow. I-DEAS, SDRC, 1999.

[36] NC Machining. Pro/Engineer, 1999.

[37] “NURBS Modeling”, Studio 9 Manual, Alias|Wavefront, 1999.

[38] Powerflow, 1999: (http:///www.rand.com/).

[39] Proceedings of the Seventh Symposium on Construction Robotics in Japan, 1998 (http://www.jssst.or.jp/jsce2/committee/robot/sinpo7.htm).

[40] Proxim. RangeLAN2, 1999: (http://www.proxim.com/).

[41] Ross, J. The ERP Revolution: Surviving Versus Thriving. Research Paper. Cambridge: Center for Information Systems, MIT, 1999.

[42] Simon, H.A. Administrative behavior. New York: Macmillan Co., 1947.

[43] U.S. Congressional Board. Advanced materials by design. Washington DC: Office of Technological Assessment, 1988.

[44] Vericut, 1999: (http://www.vericut.com/).

[45] VirtualNC, 1999: (http://www.deneb.com/).

[46] Willcocks, L. Feeny, D. Islei, G. Managing IT as a Strategic Resource. Maidenhead: McGraw-Hill, 1997.

[47] Zermani, A. Goh, HCC. "Finite element analysis of timber joints incorporating mechanical connectors", Topping B.H.V. and Papadrakakis M (Eds.), Advances in Structural Engineering Computing. Edinburgh: Civil-Comp press, 1994.

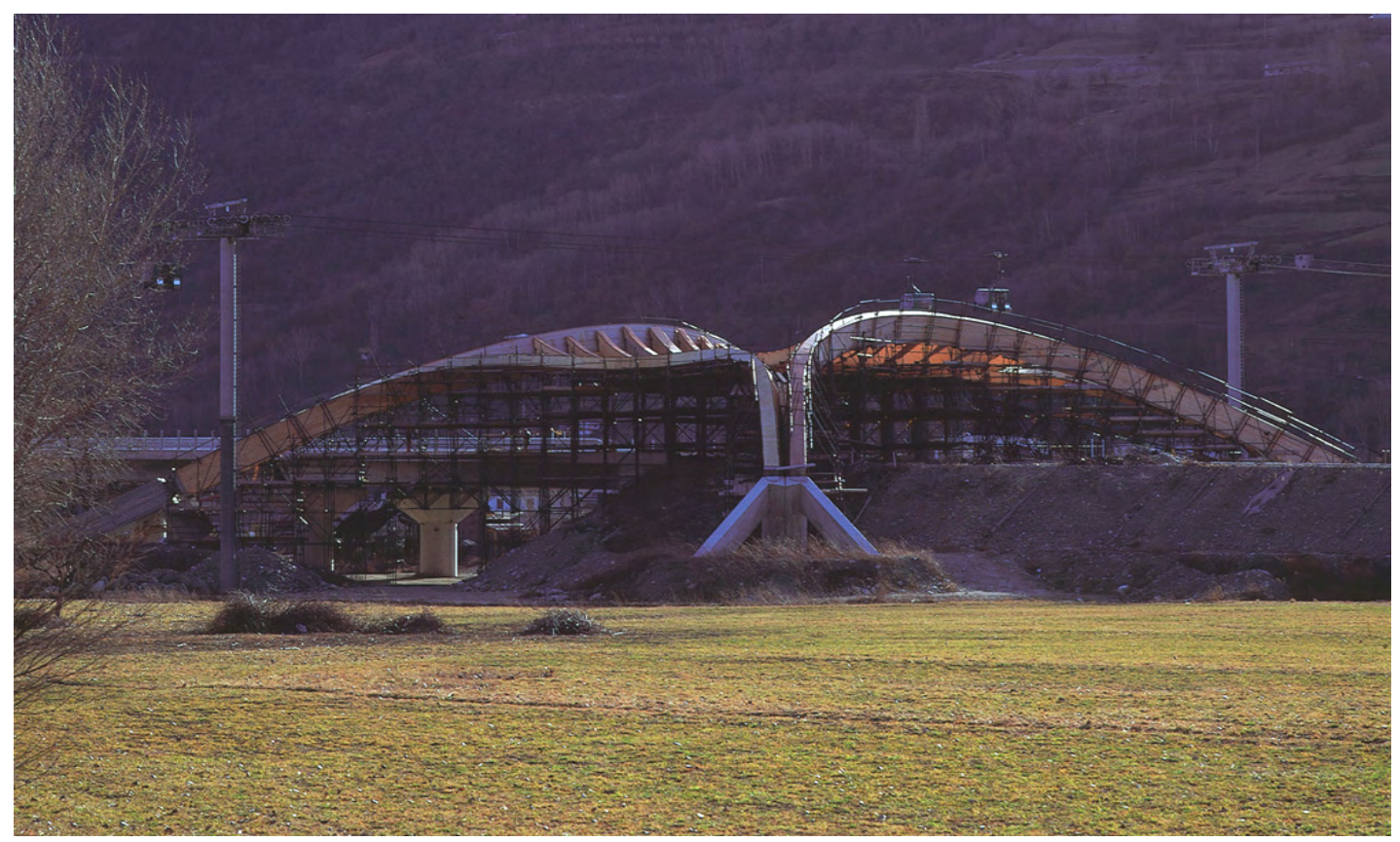

Figure 13. The structure completed with the temporary scaffolding still up. 